\title{
Color-Weakness Compensation using Riemann Normal Coordinates
}

\author{
Satoshi Oshima, Rika Mochizuki, Reiner Lenz and Jinhui Chao
}

\section{Linköping University Post Print}

N.B.: When citing this work, cite the original article.

C2012 IEEE. Personal use of this material is permitted. However, permission to reprint/republish this material for advertising or promotional purposes or for creating new collective works for resale or redistribution to servers or lists, or to reuse any copyrighted component of this work in other works must be obtained from the IEEE.

Satoshi Oshima, Rika Mochizuki, Reiner Lenz and Jinhui Chao, Color-Weakness Compensation using Riemann Normal Coordinates, 2012 IEEE International Symposium on Multimedia.

http://dx.doi.org/10.1109/ISM.2012.42

Postprint available at: Linköping University Electronic Press

http://urn.kb.se/resolve?urn=urn:nbn:se:liu:diva-86669 


\section{Color-Weakness Compensation using Riemann Normal Coordinates}

$\begin{array}{cccc}\text { Satoshi Oshima } & \text { Rika Mochizuki } & \text { Reiner Lenz } & \text { Jinhui Chao } \\ \text { Chuo University } & \text { Chuo University } & \text { Linköping University } & \text { Chuo University } \\ \text { NTT Advanced Tech. } & \text { Tokyo, Japan } & \text { Norrköping, Sweden } & \text { Tokyo, Japan } \\ \text { Tokyo, Japan } & \text { mochizuki.rika@chao.ise.chuo-u.ac.jp } & \text { Reiner.Lenz@liu.se } & \text { jchao@ise.chuo-u.ac.jp } \\ \text { soshima@chao.ise.chuo-u.ac.jp } & & & \end{array}$

\begin{abstract}
We introduce normal coordinates in Riemann spaces as a tool to construct color-weak compensation methods. We use them to compute color stimuli for a color weak observers that result in the same color perception as the original image presented to a color normal observer in the sense that perceived color-differences are identical for both. The compensation is obtained through a color-difference-preserving map, i.e. an isometry between the 3D color spaces of a colornormal and any given color-weak observer. This approach uses discrimination threshold data and is free from approximation errors due to local linearization. The performance is evaluated with the help of semantic differential (SD) tests.
\end{abstract}

Keywords-Human-computer interaction, universal design, color-weak compensation; discrimination threshold; semantic differential;

\section{INTRODUCTION}

The properties human color vision vary widely between individuals. This is obviously the case for observers with normal color vision and color blind persons, but there are also significant differences between observers with normal color vision. There are also a significant number of persons with color-weak color vision. It is therefore important to be able to adapt the presentation of visual information such that variations in color vision are compensated. This is one of the most important and challenging tasks in universal design and barrier-free IT technology.

Solving this problem is very hard since color vision is a very complex process whose mechanisms are largely unknown. Even in the sensor-related, low-level side we cannot measure quantitative properties of human perception with objective and non-invasive methods and it is therefore impossible to characterize the color-weakness of an observer exactly. This is the reason why there are, up to now, no methods to compensate color defects even on the basic signal processing level.

Given the lack of such direct measurements we followed the standard procedure in color science to measure one of the fundamental color vision data, the discrimination thresholds and develop an algorithm for color-weakness compensation by making a novel use of these data (see [3], [6], [7]). The method is based on the requirement that the color-weak observer should experience the same color differences as average color normal observers. Technically this is achieved by matching discrimination thresholds of the color-weak and the color-normal observer average based on the Riemann geometric properties of color spaces. These small color differences or discrimination thresholds (known as the MacAdam ellipsoids) are among the few observables in color perception which can be used to characterize the perceptual characteristics of individual observers. Such a discrimination ellipsoid describes the just-noticeable difference between the color at the center of the ellipsoid and other colors in its neighborhood. In the Riemann geometry framework these ellipsoids characterize the local geometry around center points, and the color space becomes a Riemann space with the thresholds defining the Riemann metric. Then a global compensation can be obtained by integrating these local data using tools from Riemann geometry. A recent evaluation of this approach is reported in [9]. For special types of color weakness, closed form, 1-D compensation methods and their fast implementation are described in [7]. For many color-weak observers these methods are not general enough and simultaneous compensations in more than one dimension are necessary. There is thus a need for $2 \mathrm{D}$ and $3 \mathrm{D}$ methods like the ones based on local affine maps which are described in [3] and [6]. These mappings apply techniques from linear algebra and they are therefore easy to implement. Unfortunately their perfomance is limited due to the approximation errors originating in the local linearization.

In this paper we present a compensation without local linearization based on the global Riemann geometry of 3D color spaces to avoids these errors. In [5] discrimination threshold data were measured on the chromaticity plane of the CIEXYZ space and a 2D compensation was implemented. The experiments showed that more accurate threshold data were required and that chromaticity information alone was, in general, not sufficient to produce satisfying compensation results. This showed that a simultaneous compensation of both lightness and chromaticity in 3D color spaces is needed. In this paper we build on these results by first presenting an explicit and systematic description of a compensation principle based on global color difference preserving maps or isometries. Furthermore, we report on a new set of discrimination threshold measurements for both 
color-weak and normal observers in CIELUV space of reasonably high resolution. Based on these results we construct uniform color spaces of color-weak and normal observers using Riemann normal coordinates in both color spaces. This results in a global isometry which is then applied for colorweak simulation and its inverse, the compensation map. The proposed method is applied to natural images using measured discrimination thresholds of colorweak and normal observers. Its performance is evaluated using semantic differential (SD) tests [8].

\section{COMPENSATION AND THE RIEMANN GEOMETRY OF COLOR SPACES}

A Riemann space is a space with a local geometry described by the metric tensor $G(x)$ at a point $x$ ([1], [2]). In particular the distance between $x$ and a point in its neighborhood located at a distance $\Delta x$ from it is, approximately, given by:

$$
\|\Delta x\|^{2}=\Delta x^{T} G(x) \Delta x .
$$

The local metric, given by the positive-symmetric matrix $G(x)$ is derived from the just-noticeable color differences in the color spaces. The differences define the MacAdam ellipses and ellipsoids. They are among the first measurements of the metric tensor in 2D and 3D spaces and color vision was one of the application areas mentioned by Riemann when he introduced his new geometrical construction. The distance $d\left(x_{1}, x_{2}\right)$ between two points $x_{1}, x_{2}$ in a Riemann space is defined as the length of the shortest path $\gamma_{12}$ connecting the two points. These paths are known as geodesics and large color differences in color spaces can also be defined in this manner:

$$
d\left(x_{1}, x_{2}\right)=\int_{\gamma_{12}}\|\Delta x\|=\int_{\gamma_{12}} \sqrt{\Delta x^{T} G(x) \Delta x} .
$$

We model the color perception of an individual observer by deriving the metric of the color space from his/her threshold measurements. Subjective color differences are then measured as distances in this color space. Constructing colordifference-preserving maps $f$ between different observers is then reduced to find isometries from space $C_{1}$ with Riemann metric $G_{1}(x)$ to space $C_{2}$ with metric $G_{2}(y)$ such that

$$
G_{1}(x)=\left(D_{f}\right)^{T} G_{2}(y) D_{f},
$$

where $D_{f}$ is the Jacobian of $f$. This is a local isometry which always maps the threshold ellipsoid at $x$ onto the threshold ellipsoid at $y=f(x)$. It can be shown that this also gives a global isometry where the distances between $y_{1}=f\left(x_{1}\right)$ and $y_{2}=f\left(x_{2}\right)$ are always the same as that between $x_{1}$ and $x_{2}$. For details using the local isometries see [3] and [6].

Here we construct a global isometry directly from Riemann normal coordinates. These coordinates correspond to polar coordinates in Euclidean space and are constructed as

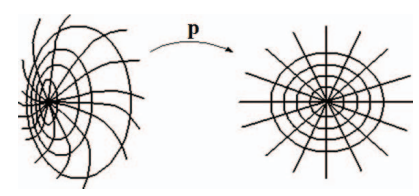

Figure 1: Coordinate transformation

follows: From a given point, the origin, one draws geodesics of a given length which are all separated by the same angle. Then one connects the points on adjacent geodesics to obtain the Riemann normal coordinates. In this way an isometry $p$ between the color space and the Euclidean space is obtained. This Euclidean space corresponds to the uniform color space $U$ of the original color space and $p$ is called a uniformization map (see Fig.1) Now given two color spaces $C_{1}$ and $C_{2}$, we draw from a common origin geodesics to obtain two Riemann normal coordinates, one in each color space. Denote the uniformization from $C_{i}$ to $U$ as $p_{i}, i=1,2$. Then since the inverse of an isometry and composition of two isometries are also isometry, we obtain an isometry between $C_{1}$ and $C_{2}$ as $f=p_{2}^{-1} \circ p_{1}$. Let $C_{1}, C_{2}$ be the color spaces of normal and color-weak observers, then $f$ is the color-weak simulation map, which shows to normal observers what a color-weak observer actually sees. The compensation map is defined as the inverse map of $f$, $f^{-1}=p_{1}^{-1} \circ p_{2}$.

In the following we illustrate this construction based on our measurements. We choose as the common origin the location of the D65 light source and draw geodesics which are obtained as solution curves of the following ODE:

$$
\frac{d^{2} u^{i}}{d s}+\Gamma_{j k}^{i} \frac{d u^{j}}{d s} \frac{d u^{k}}{d s}=0
$$

where $\Gamma_{j k}^{i}$ is the Christoffel symbol defined as follows:

$$
\Gamma_{j k}^{i}=\frac{1}{2} g^{i \alpha}\left(\frac{\partial g_{\alpha j}}{\partial u^{k}}+\frac{\partial g_{\alpha k}}{\partial u^{j}}-\frac{g_{j k}}{\partial u^{\alpha}}\right) .
$$

Here the metric and its inverse are denoted by $G=$ $\left(g_{i j}\right), G^{-1}=\left(g^{i j}\right)$ and the Einstein summation convention is used.

\section{DISCRIMINATION THRESHOLD MEASUREMENT}

In fact, the proposed algorithm can compensate between arbitrary two observers as long as their discrimination threshold data are available. In our experiments we measured color discrimination thresholds of a normal observer and a most typical color-weak observer of $D$ type with the adjustment method in CIELUV sRGB space and data from an Anomalouscope. We obtained 77 sampling points, at 5 different lightness levels $\mathrm{L}=30,40,50,60$, 70. Data grids in the chromaticity planes of these levels contain 9, 13, 19, 20, 16 points. An ellipsoid at a point is estimated from deviations in 14 directions from the point. We used 


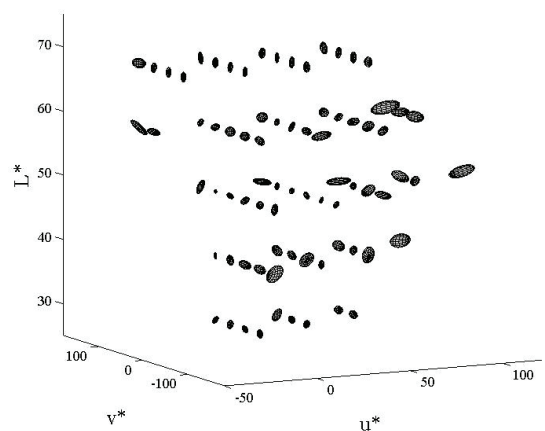

(a) The color-normal observers

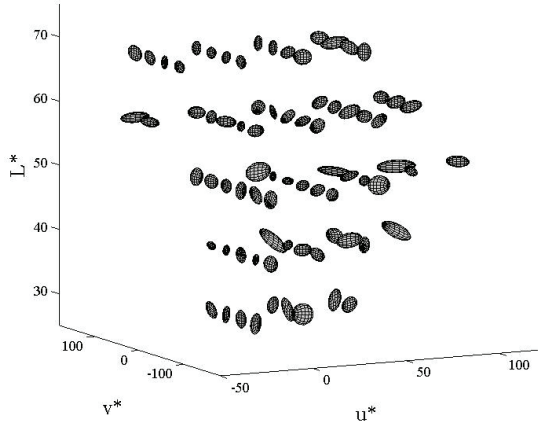

(b) The color-weak observer

Figure 2: Discrimination ellipsoids

SyncMaster XL24 monitor by Samson illuminated by Panasonic Hf PREMIER fluorescent lamps, with Munsell N5.5 background and the 10 degree view angle.

The ellipsoids of the normal and the color-weak observer are shown in Figs. 2a, 2b. The Riemann normal coordinates in $L=50$ for normal and color-weak observers are shown in Figs.3aand 3b. The Riemann normal coordinates in CIELUV space are shown in Figs.4a and 4b. For better visibility only geodesics are shown. Compared with the normal observer an obvious distortion in Riemann normal coordinates of the color-weak observer can be observed which can not be described by $1 \mathrm{D}$ distortions along confusion lines. Thus precise representation of color vision characteristics can only be provided by Riemann normal coordinates in higher dimensions.

\section{AppliCATION AND EVALUATION}

We illustrate the algorithm by constructing a compensation map between the color-normal and color-weak observers. The original image is shown in Fig.5a and the 2D and 3D color-weak compensations are shown in Figs. 5b and $5 \mathrm{c}$. It can be observed that the $2 \mathrm{D}$ compensation enhanced both red and green but with quite different $\mathrm{L}$ values. Meanwhile, the 3D compensation combined chromaticity with lightness to produce more natural tone and contrast and therefore a balanced color distribution.

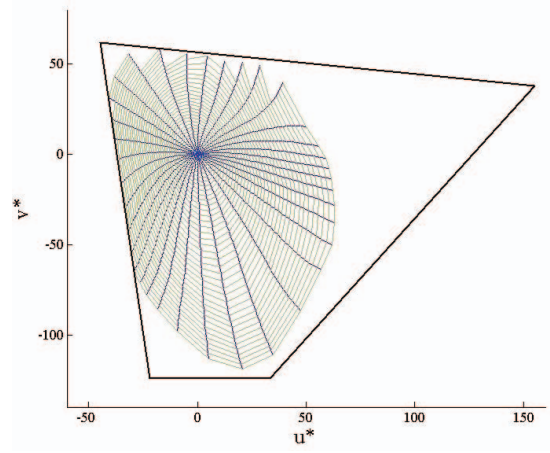

(a) The color-normal observers at $\mathrm{L}=50$

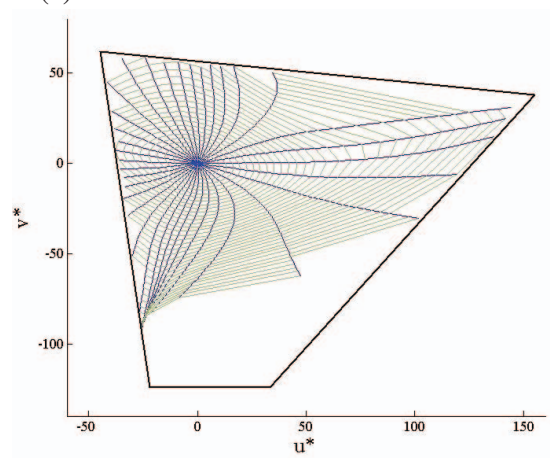

(b) The color-weak observer at $\mathrm{L}=50$

Figure 3: 2D Riemann normal coordinates

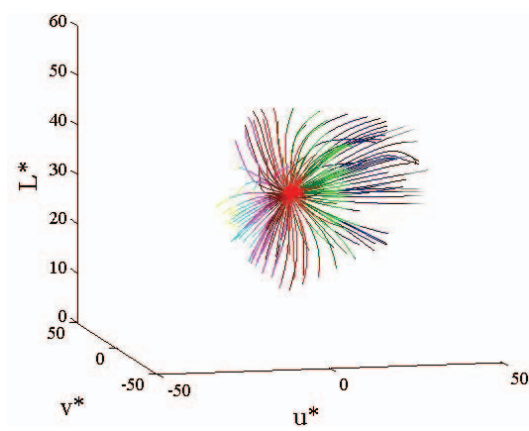

(a) The color-normal observers

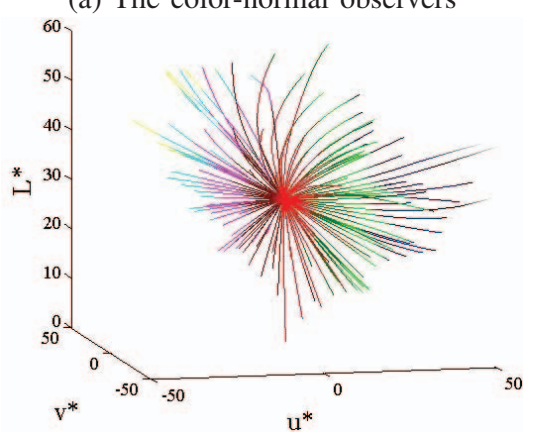

(b) The color-weak observer

Figure 4: 3D Riemann normal coordinates 


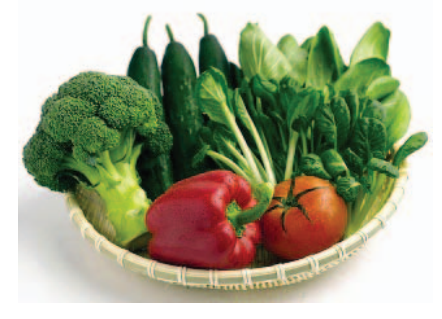

(a) Original

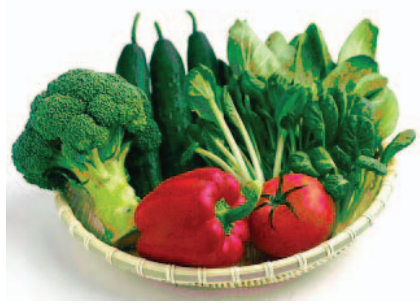

(b) $2 \mathrm{D}$ compensation

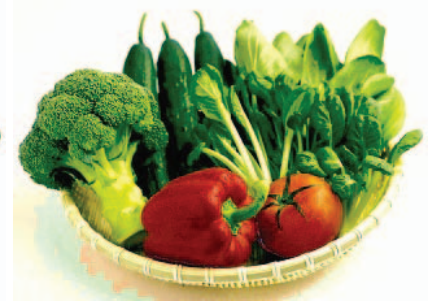

(c) 3D compensation
Figure 5: Images

Since a direct evaluation of the perfomance of compensation is difficult, especially for natural images with complicated color distributions, we used the semantic differential method [8] to compare visual impression before and after compensation. 20 adjectives as given by Osgood were used with a seven scales questionnaire. The results and the correlation coefficients between SD scores of a color normal observer seeing the original and a colorweak observer seeing the compensated images are shown in Table I. These results indicate that the compensation leads to a closer similarity between the perception of the color-weak and the normal observer.

\section{SUMmary}

We introduced Riemann normal coordinates as a tool to construct mappings between the color spaces with metrics defined by the perception properties of different observers. These metrics were measured for colorweak and color normal observers and 2D and 3D color-weak compensation were implemented. The semantic differential evaluation showed that both the 2D and the 3D compensations increased correlation between impressions of the color normal and the colorweak observer and that the $3 \mathrm{D}$ compensation is more efficient than the $2 \mathrm{D}$ compensation.

\section{Acknowledgment}

This research is partially supported by Grant-in-Aid for Scientific Research of MEXT and JSPS Japan, the Institute of Science and Engineering, Chuo University and the Swedish Foundation for Strategic Research through grant IIS11-0081. The research leading to these results has also received funding from the European Community's Seventh Framework Programme FP7/2007-2013 - Challenge 2

\begin{tabular}{|c|c|c|c|c|}
\hline \multirow{2}{*}{$\begin{array}{c}\text { adjectives } \\
\text { score:0 - score: }\end{array}$} & \multicolumn{4}{|c|}{ score } \\
\cline { 2 - 5 } & \multicolumn{2}{|c|}{ original } & 2D & $3 \mathrm{D}$ \\
\cline { 2 - 5 } large - small & 5 & 5 & 6 & 5 \\
\hline simple - complex & 3 & 5 & 5 & 4 \\
\hline intangible - tangible & 6 & 2 & 3 & 4 \\
\hline cruel - kind & 6 & 5 & 5 & 5 \\
\hline passive - active & 4 & 2 & 3 & 5 \\
\hline wet - dry & 3 & 3 & 2 & 3 \\
\hline strong - weak & 5 & 4 & 2 & 3 \\
\hline light - heavy & 3 & 5 & 5 & 5 \\
\hline youthful - mature & 6 & 3 & 5 & 5 \\
\hline opaque - transparent & 4 & 2 & 3 & 2 \\
\hline blunt - sharp & 3 & 3 & 2 & 3 \\
\hline masculine - feminine & 6 & 5 & 5 & 6 \\
\hline regressive - progressive & 3 & 3 & 5 & 4 \\
\hline excitable - calm & 5 & 4 & 3 & 3 \\
\hline near - far & 3 & 2 & 2 & 3 \\
\hline soft - hard & 2 & 3 & 3 & 3 \\
\hline ornate - plain & 2 & 3 & 2 & 3 \\
\hline cold - hot & 3 & 2 & 2 & 2 \\
\hline intuitive - rational & 3 & 3 & 2 & 2 \\
\hline constrained - free & 5 & 2 & 2 & 3 \\
\hline compensation type & without & after $2 \mathrm{D}$ & after $3 \mathrm{D}$ \\
\hline correlation coefficient & 0.227927 & 0.375807 & 0.495615 \\
\hline
\end{tabular}

Table I: SD scores and correlation coefficients

- Cognitive Systems, Interaction, Robotics - under grant agreement No 247947 - GARNICS.

\section{REFERENCES}

[1] G. Wyszecki, W.S. Stiles "Color Science", Wiley, 2000.

[2] M. P. do Carmo, "Riemannian Geometry", Birkhauser, 1992.

[3] R.Mochizuki, T.Nakamura, J.Chao, R.Lenz, "Colorweak correction by discrimination threshold matching", Proc.CGIV2008, pp.208-213, IS\&T, 2008.

[4] J.Chao, R.Lenz, D.Matsumoto, T.Nakamura,'Riemann framework for color characterization and mappings," Proc. CGIV2008, pp.277-282, IS\&T 2008.

[5] S.Oshima, R.Mochizuki, J. Chao, and R. Lenz, "Color reproduction using Riemann normal coordinates," Proc. Comp. Color Imaging Workshop, LNCS-5646, pp.140-149, 2009.

[6] R.Mochizuki, S.Oshima, R.Lenz, J.Chao "Exact compensation of color-weakness with discrimination threshold matching" HCI International 2011 Conference, LNCS-6768, pp.155-164, 2011.

[7] R.Mochizuki, S.Oshima, J.Chao "Fast color-weakness compensation with discrimination threshold matching" "Computational Color Imaging ”, LNCS-6626, pp.176-187, 2011.

[8] E. Osgood, GJ. Suci, and Percy H. Tannenbaum, "The Measurement Of Meaning," pp.53-61, University of Illinois, Urbana, Chicago, and London, 1957.

[9] Y.C.Chen, Y.Takahashi, Y.Guan, T.Ishikawa, H.Eto, T.Nakatsue, J.Chao, M.Ayama "KANSEI Evaluation of Color Images Corrected for Color Anomalies assessed by Deuteranomalous and Normal Observers" Proc. 17th International Display Workshop,2010. 